\title{
Teste da linguinha: as gestantes sabem do que se trata?
}

\author{
Tongue test: the pregnant women know what it is?
}

Teste de la lengua: las gestantes saben de que se trata?

\begin{abstract}
Elizandra Silva da Penha ${ }^{1 *}$, Ana Karina Almeida Rolim², Priscila Oliveira das Chagas ${ }^{1}$, Laísa Pereira Ribeiro ${ }^{1}$, Gymenna Maria Tenório Guênes ${ }^{1}$, Luanna Abílio Diniz Melquíades de Medeiros ${ }^{1}$, Rosana de Araújo Rosendo', Camila Helena Machado da Costa Figueiredo¹.
\end{abstract}

\section{RESUMO}

Objetivo: Determinar o conhecimento de gestantes atendidas nas Unidades Básicas de Saúde da Família em um município do interior da Paraíba sobre o teste da linguinha. Métodos: Foi realizada pesquisa coorte transversal, com 275 gestantes de idade entre 15 e 34 anos. O instrumento utilizado foi um questionário composto por dez questões, onde foi indagado sobre idade e grau de escolaridade, o conhecimento ou não do teste, o que é detectado através dele, se foi orientada a realizá-lo e em casos positivos por qual profissional, quando deve ser feito, se considera importante e por qual motivo. Resultados: Os resultados apontaram um alto percentual de gestantes que não conheciam o teste $(80 \%)$ e que não foram orientadas por nenhum profissional a realizá-lo $(88,36 \%)$, porém entre os profissionais que haviam orientado, os que merecem destaque são o cirurgião-dentista $(59,37 \%)$, seguido do enfermeiro (34,37\%). Sobre quando o teste deve ser realizado, observou-se que a grande maioria (75\%) respondeu que deve ser realizado quando bebê. Conclusão: Há necessidade de promover ações educativas e preventivas através do pré-natal odontológico para que as gestantes sejam informadas sobre o teste da linguinha e procurem os locais que realizem o mesmo.

Palavras-chave: Saúde bucal, Cuidado pré-natal, Freio lingual.

\begin{abstract}
Objective: Determine the knowledge of pregnant women that were monitored at health centers in the interior of Paraíba about such test. Methods: A cross-sectional observational study was conducted, with 275 pregnant women ate the age of 15 to 34 years old. The instrument used was a composed questionnaire of ten questions, which was questioned about the age and schooling level, the knowledge of the test or not, what is detected through it, if she was oriented to do it and, if yes, which professional did it, when it should be done, if she considered that important and for what reason. Results: The results showed a high percentage of pregnant women who did not know the test $(80 \%)$ and who were not guided by any professional to perform it $(88.36 \%)$, but among the professionals who instructed, the ones who are noteworthy are the dentists $(59.37 \%)$, followed up by nurses (34.37\%). Regarding about when the test should be performed, it was observed that the majority $(75 \%)$ answered that it should be performed when the child is a baby. Conclusion: There is a need to promote educational and preventive actions through prenatal dentistry so that pregnant women are informed about the tongue test and look for places that perform the same.
\end{abstract}

Key words: Oral health, Prenatal care, Lingual frenum.

1 Universidade Federal de Campina Grande (UFCG), Patos, Paraíba. *E-mail: elizandrapenha@hotmail.com

2 Universidade Estadual da Paraíba (UEPB), Campina Grande, Paraíba.

SUBMETIDO EM: 5/2019

ACEITO EM: 6/2019

PUBLICADO EM: 7/2019

REAS/EJCH | Vol. 11(13) | e957 | DOI: https://doi.org/10.25248/reas.e957.2019 Página 1 de 7 


\section{RESUMEN}

Objetivo: Determinar el conocimiento de gestantes atendidas en las Unidades Básicas de Salud de la Familia en un municipio del interior de Paraíba sobre la prueba de la lengua. Métodos: Se realizó un estudio observacional transversal., con 275 gestantes de edad entre 15 y 34 años. El instrumento utilizado fue un cuestionario compuesto por 10 preguntas, siendo 7 cuestiones y 3 cuestiones donde fue indagado sobre edad y grado de escolaridad, el conocimiento o no del test, lo que es detectado a través de él, si se orientó a realizarlo y en casos positivos por cual profesional, cuando debe hacerse, se considera importante y por qué motivo. Resultados: Los resultados apuntaron un alto porcentaje de gestantes que no conocían la prueba $(80 \%)$ y que no fueron orientadas por ningún profesional a realizarlo $(88,36 \%)$, pero entre los profesionales que habían orientaron a los que merecen destaque, cirujano-dentista $(59,37 \%)$, seguido del enfermero $(34,37 \%)$. Sobre cuando la prueba debe ser realizada, se observó que la gran mayoría (75\%) respondió que debe ser realizado cuando el bebé. Conclusión: Hay necesidad de promover acciones educativas y preventivas a través del prenatal odontológico para que las gestantes sean informadas sobre la prueba de la lengua y busquen los lugares que realicen el mismo.

Palabras clave: Salud bucal, Ateción prenatal, Frenillo lingual.

\section{INTRODUÇÃO}

A língua é um órgão com participação direta nas atividades de deglutição, mastigação, sucção e fala, que estão diretamente relacionadas com a respiração (BRAGA LAS et al., 2009).

Possui em sua face inferior uma pequena prega de membrana mucosa que a conecta ao assoalho da boca, denominada de frênulo. Este possibilita ou interfere a livre movimentação desta estrutura (BRASIL, 2013).

Anquiloglossia é uma anomalia do desenvolvimento caracterizada por alteração no frênulo lingual e seu diagnóstico é feito baseado no conhecimento profundo da anatomia da língua e das áreas adjacentes (BRASIL, 2013; BRASIL, 2014).

Esta anormalidade dificulta os movimentos desse órgão e traz como consequência para os recémnascidos, a amamentação inadequada, pouco ganho de peso e desmame precoce e para as mães dor e desconforto (BRITO SF et al., 2008).

Caso esta alteração não seja tratada precocemente, a criança pode apresentar problemas futuros que podem implicar em constrangimentos sociais, como a dificuldade de articulação da fala, dificuldade de lamber os lábios e sorvetes, por exemplo (CARVALHO CM et al., 2013).

Em recém-nascidos e lactantes a frenotomia é o tratamento cirúrgico de escolha da anguiloglossia, e consiste na incisão linear anteroposterior do frênulo lingual, sem remoção do tecido. Outro procedimento cirúrgico que pode ser realizado é a frenectomia, que se dá através da remoção completa do tecido mucoso que compõem o frênulo lingual. A escolha de qual procedimento realizar dependerá da idade da intervenção, bem como da espessura do freio em questão (CONSOLARO A et al., 2014).

Martinelli RLC et al. (2012) desenvolveram um protocolo de avaliação do frênulo da língua para bebês denominado teste da linguinha, com a finalidade de identificar se este limita os movimentos da mesma. A obrigatoriedade da realização desse protocolo em todos os hospitais e maternidades do Brasil se deu a partir da lei número 13.002 de 20 de junho de 2014 que fez com que o país se tornasse o primeiro a oferecer este teste (GARCIA OJ et al., 2008).

Segundo a lei, o teste deve ser feito por um profissional de saúde habilitado e ser realizado seguindo o protocolo de avaliação que é dividido em história clínica, avaliação anatomofuncional e avaliação da sucção nutritiva e não nutritiva. Preferencialmente o teste deve ser realizado durante o primeiro mês de vida (GARCIA OJ et al., 2008). 
A gestante possui diversas necessidades durante a gravidez, o que inclui às informações relativas à saúde geral, bem como, da saúde bucal. Assim, durante o pré-natal, tais orientações devem ser repassadas, a fim de prepará-la para receber seu filho. O cirurgião dentista deve fazer parte da equipe de saúde do pré-natal, realizando ações ao nível da promoção da saúde, como orientações sobre hábitos de higiene, cuidados com a saúde bucal do seu bebê e em outros níveis de prevenção, mais ligados à atenção à doença (GENARO KF et al., 2009).

Cabe à equipe orientar sobre a importância do teste e o aleitamento materno de forma segura e tranquila durante o pré-natal. Com a realização do teste é possível diagnosticar e tratar precocemente a anquiloglossia, evitando desta maneira a amamentação inadequada e o desmame precoce. $\mathrm{O}$ abandono à amamentação natural expõe o recém-nascido à maior risco de morte e doenças, decorrentes da perda de fatores de proteção encontrados no leite humano. Geralmente o neonato passa a fazer uso de mamadeiras, o que pode causar hábitos de sucção deletérios que levam a deformação da arcada dentária e o surgimento de respiração bucal. Diante do exposto, esse trabalho tem como objetivo avaliar o conhecimento das gestantes atendidas nas Unidades Básicas de Saúde da Família (UBSF's) acerca do teste da linguinha.

\section{MÉTODOS}

Foi realizado um estudo do tipo quantitativo, de coorte transversal, com abordagem indutiva e aprovado pelo Comitê de Ética em Pesquisa com Seres Humanos das Faculdades Integradas de Patos (Certificado de Apresentação e Apreciação Ética - CAAE: 59871916.9.0000.5181).

O estudo foi desenvolvido em 42 UBSF's, localizadas no município de Patos, situado no alto sertão paraibano. Antes do início da pesquisa, foram explicados seus objetivos e sua importância, utilizando-se de uma linguagem simples, direta e de acordo com o nível de escolaridade e idade de cada gestante. Quando as mesmas concordaram em participar da pesquisa, foram convidadas a assinar o Termo de Consentimento Livre e Esclarecido (TCLE).

A amostra de conveniência foi composta de 275 gestantes, que realizavam pré-natal obstétrico nas UBSF'S do município, onde deveriam ter realizado no mínimo três consultas, número suficiente para terem sido orientadas quanto ao teste. Foram excluídas as gestantes que não responderam o questionário de forma completa e as que apresentaram alguma deficiência congnitiva e/ou intelectual que possibilitasse a aplicação do mesmo.

O instrumento utilizado para avaliar o conhecimento sobre o teste da linguinha foi uma adaptação do questionário utilizado por Jardim JB e Hahn GV (2014). O mesmo foi composto por 10 questões, sendo 7 objetivas e 3 questões subjetivas onde foi indagado sobre idade e grau de escolaridade, o conhecimento ou não do teste, o que é detectado através dele, se foi orientada a realizá-lo e em casos positivos por qual profissional, quando deve ser feito, se considera importante e por qual motivo.

A aplicação do questionário foi feita na sala de espera das UBSF's antes das gestantes serem atendidas, fornecendo explicações básicas de como deveria ser respondido. Em casos de gestantes que não sabiam ler, as perguntas foram feitas pelo pesquisador e a mesma preenchia o questionário. Os resultados obtidos foram registrados em fichas individuais para posterior tabulação e análise. Fez-se uso dos recursos da planilha de cálculo do Programa Microsoft Excel® versão 14.0 (Office 2010).

\section{RESULTADOS}

Quanto à caracterização da amostra foram entrevistadas 275 gestantes, com maior frequência de idade entre 20 a 24 anos, somando $32 \%$, seguida de mulheres com idade de 25 a 29 anos (24,36\%), 15 a 19 anos com 20,72\%, 30 a 34 anos com 18,54\% e acima de 34 anos com apenas 4,36\%. Quanto à escolaridade, o ensino fundamental completo destacou-se com $32 \%$ da amostra, seguido de ensino fundamental incompleto com $26,90 \%$ e do ensino médio completo $24,72 \%$, ficando $5,81 \%$ com ensino médio incompleto, $5,81 \%$ com ensino superior completo e $4,36 \%$ com ensino superior incompleto (Tabela 1). 
Com relação ao conhecimento sobre o teste da linguinha, $80 \%$ das gestantes questionadas relataram nunca terem ouvido falar e apenas $20 \%$ afirmaram saber do que trata o teste. Indagadas sobre a alteração que é detectada através do teste $27,27 \%$ responderam não saber o que é detectado e $72,72 \%$ relataram saber o que o mesmo detecta (Tabela 1).

Tabela 1 - Resultados da aplicação do questionário sobre o Teste da linguinha. Patos-PB 2017.

VARIÁVEL

1. Qual sua idade?

15 a 19

20 a 24

25 a 29

30 a 34

acima de 34

2. Qual seu grau de escolaridade?

Analfabeta

Ensino fundamental completo

Ensino fundamental incompleto

Ensino médio completo

Ensino médio incompleto

Ensino superior completo

Ensino superior incompleto

3. Você já ouviu falar sobre o teste da linguinha?

Não

Sim

4. Você sabe o que o teste detecta?

Não

Sim

5. O que o teste detecta?

Língua presa

Não sei

Condição que impede a criança de falar e comer direito

Condição que impede a criança de falar corretamente

Já ouvi falar, para que serve não sei

Alteração do freio lingual

6 . Foi orientada a realizar o teste?

Não

Sim

7. Qual profissional orientou a realizar o teste?

Dentista

Enfermeiro

Agente de saúde

Médico

8. Quando o teste deve ser realizado?

Quando bebê

Não sei

Após nascimento

Nos primeiros 30 dias

9. Considera importante realizá-lo?

Não sei informar

Sim

10. Por qual motivo considera importante realizá-lo?

Diagnosticar um problema de alimentação

Diagnosticar e tratar precocemente

Evitar problemas sociais para criança

Todas certas

Não sei informar

Evitar futuras complicações

Fonte: Dados da pesquisa, 2017.

\section{$\mathrm{N}$}

57

88

67

51

12

1

88

74

68

16

16

12

220

55

15

40

34

2

1

1

1

1

243

32

19

11

1

1

24

4

2

2

$6,25 \%$

$11 \quad 20 \%$

$44 \quad 80 \%$

$23 \quad 52,27 \%$

$10 \quad 22,72 \%$

$5 \quad 11,36 \%$

$3 \quad 6,81 \%$

$2 \quad 4,54 \%$

1
$20,72 \%$

$24,36 \%$

$18,54 \%$

$4,36 \%$

$0,36 \%$

$26,90 \%$

$4,72 \%$

$5,81 \%$

$4,36 \%$

$80 \%$

$27,27 \%$

$72,72 \%$

$85,00 \%$

$2,50 \%$

$2,50 \%$

$2,50 \%$

$2,50 \%$

$88,36 \%$

$11,63 \%$

$34,37 \%$

$3,12 \%$

$3,12 \%$

$75,00 \%$

$2,50 \%$

$6,25 \%$ $2,27 \%$ 
Quanto a serem orientadas a realizarem o teste, $88,36 \%$ não receberam nenhuma orientação e somente $11,63 \%$ responderam positivamente. Dentre os profissionais que orientaram, o dentista foi citado por $59,37 \%$ como o profissional que mais realizou orientações, seguido do enfermeiro com $34,37 \%$ das respostas e médico e agente de saúde com apenas $3,12 \%$, uma resposta cada (Tabela 1).

As gestantes que afirmaram ser importante realizar o teste correspondem a $80 \%$ da amostra e uma porcentagem de $20 \%$ afirmaram não saber informar. Em relação ao motivo pelo qual consideram importante, $52,27 \%$ responderam que por diagnosticar um problema na alimentação, 22,72\% para diagnosticar e tratar precocemente, 11,3 para evitar problemas sociais para criança, 4,54\% não sabiam, $6,81 \%$ responderam todas as alternativas como importante e apenas $2,27 \%$ respondeu para evitar futuras complicações (Tabela 1).

\section{DISCUSSÃO}

A gestação é o período no qual a mulher se mostra mais receptiva a adquirir conhecimentos que possam ser revertidos em benefício ao seu bebê. Desse modo, as atitudes e escolhas maternas certamente refletirão no desenvolvimento de um bebê saudável. Gestantes devem ser tratadas como grupo prioritário onde ações educativas e preventivas devem ser realizadas através do pré-natal odontológico para que a mesma cuide da saúde bucal do seu filho e previna possíveis complicações (GENARO KF, et al. 2009).

O pré-natal odontológico além de garantir a assistência do tratamento das doenças bucais, possui um perfil informativo e esclarecedor quanto aos cuidados básicos de higiene, alimentação, suplementação de flúor e cálcio, hábitos deletérios, dentre outros que possam interferir na saúde do futuro bebê (HOCHNADEL DB, 2011).

O estudo em questão procurou avaliar o conhecimento das gestantes que realizaram pré-natal odontológico nas UBSF's a respeito do teste da linguinha, onde foi constatado que a porcentagem de puérperas que nunca ouviram falar do teste é elevada e a grande maioria das mesmas afirmou não ter sido orientada por nenhum profissional de saúde a realizar o mesmo.

Embora considerando que algumas entrevistadas ainda iriam comparecer a consultas pré-natais, há de considerar que a equipe multiprofissional de saúde que atendeu as gestantes do estudo ainda poderia nas próximas consultas repassar informações quanto ao teste. A não ocorrência disso pode ter sido ocasionada por diversos motivos como; negligência no repasse dessas informações e desconhecimento de informações pertinentes ao teste, visto que o mesmo é um protocolo validado recentemente, com poucos anos de obrigatoriedade de ser realizado em hospitais e maternidades do Brasil. Sendo assim, existe a possibilidade dos profissionais não terem sido informados sobre o teste e nem terem recebido capacitação para realizá-lo. Carvalho CM et al. (2014) no seu estudo afirmam que a atuação da equipe de saúde no pré-natal obstétrico é extremamente importante, pois é de responsabilidade da mesma ter o conhecimento científico necessário para orientar as gestantes sobre as principais posturas a serem adotadas durante e após o período gestacional. Portanto, se faz necessário que a mesma receba atualização e capacitação para orientar as gestantes por meio de campanhas e reuniões nas maternidades e postos de saúde do Brasil sobre a significância da realização do teste da linguinha.

A faixa etária de gestantes entre 15 a 24 anos corresponde a $52,72 \%$ do total da amostra e quanto ao grau de escolaridade o ensino fundamental completo somado ao ensino fundamental incompleto e analfabetismo totalizou 59,26\% da amostra do estudo. Com isso, conclui-se que a maior parcela das participantes do estudo que não souberam responder sobre a existência do teste, o que o mesmo detecta, período ideal e a importância que é realizá-lo é composta por gestantes jovens e com baixo grau de escolaridade. Resultado semelhante foi encontrado em um estudo realizado com 413 gestantes com idades entre 15 a 44 anos acerca do conhecimento sobre a triagem auditiva neonatal. Nesse, $68,7 \%$ nunca ouviram falar do teste, $74,1 \%$ desconhecem o período ideal e 97,3\% não sabem a importância de realizá-lo. Ainda nesse estudo observouse que a faixa etária que apresentou menos respostas corretas foi a de 15 a 19 anos e quanto ao nível de escolaridade, as gestantes que apresentaram o maior número de respostas incorretas $(81,4 \%)$ foram aquelas que tinham realizado somente ensino fundamental (MARTINELLI RLC et al., 2012). 
A idade e o nível de escolaridade podem influenciar no acesso às informações, devido à provável dificuldade de leitura, escrita e interpretação de orientações repassadas. Consequentemente as chances de aprendizagem relacionadas a saúde diminui drasticamente, bem como as ações preventivas de doenças, que podem não ser valorizadas e reconhecidas por grupos jovens e de baixo grau de escolaridade (MARTINELLI RLC et al., 2013). Com relação às orientações a respeito do Teste da Linguinha, os profissionais que mais as realizaram foram o cirurgião-dentista seguido do enfermeiro. Visto que o Ministério da Saúde a respeito da organização dos serviços nas UBSF's preconiza que diversos profissionais das equipes de saúde devem integrar seus trabalhos a fim de produzir um efeito potencializador das suas ações, a interdisciplinaridade entre os profissionais em orientar, caracteriza-se como um ponto positivo (SILVA MBGM e LACERDA MR, 2003). Questionadas sobre o período ideal de realização do teste, a grande maioria das gestantes respondeu que deve ser realizado ainda quando bebê. Resultado semelhante foi obtido em estudo a respeito do conhecimento das gestantes sobre o teste do pezinho, onde $95 \%$ das mesmas responderam que o período ideal de realização do teste seria quando bebê (MEDEIROS EB e RODRIGUES MJ, 2003).

Existem protocolos como o Teste do pezinho, criado e implementado pela Portaria do Ministério da Saúde MG/MS n.o 822/01 (BRASIL, 2001), que tem como objetivo detectar e tratar precocemente doenças que, se prevenidas, evitam sequelas como a deficiência mental. Existe ainda o Teste da orelhinha, que objetiva diagnosticar e tratar precocemente deficiência auditiva (DA) e deve ser realizado preferencialmente nos primeiros seis meses de vida, período considerado crítico e ideal para a estimulação e desenvolvimento das habilidades auditivas e de linguagem. Por fim, verificou-se a necessidade de padronização da avaliação do frênulo lingual em bebês e desse modo criou-se o Teste da linguinha que tem como objetivo o diagnóstico e tratamento precoce da anquiloglossia, prevenindo assim dificuldades na amamentação, possível perda de peso e, principalmente, o desmame precoce (MELO NSF, et al., 2011; NASCIMENTO LS et al., 2015). Os protocolos de avaliação são importantes, pois permitem a padronização dos exames realizados pelos profissionais, proporcionando a comparação dos resultados obtidos com outros estudos de pesquisa, além de estabelecer parâmetros que permitam avaliar os casos de alterações e assim definir o tratamento adequado (PETERSON LJ, 2009).

Segundo o Parecer Técnico-Científico publicado pelo Instituto da saúde em 2015, o protocolo proposto por Martinelli RLC et al. (2012) contém critérios para triagem e confirmação diagnóstica, porém apresenta algumas fragilidades: difícil aplicação, a avaliação da amamentação é feita de forma subjetiva com base no relato da mãe e possui itens de observação não validados. Dentre estes itens estão a observação da mamada, que é realizada somente em 5 minutos, contradizendo recomendações da Organização Mundial de Saúde/Fundo das Nações Unidas para a Infância (OMS/UNICEF) para verificação de variáveis referentes ao início/meio/fim da mamada e os critérios de avaliação anatomo-funcional que também não foram validados 19.

Apesar disto, o teste da linguinha é o único protocolo existente para avaliar o frênulo lingual de bebês e pode ser realizado até os seis meses de vida (REIS DM, et al. 2010; SIMÃO $R$ et al., 2008). Este protocolo além de avaliar a mobilidade da língua e o frênulo, também examina estrutura e função do mesmo. Outra particularidade encontrada é a possibilidade de avaliar a relação entre anquiloglossia e a dificuldade de amamentação. O teste obrigatoriamente deve ser realizado em hospitais e maternidades, permitindo deste modo à identificação precoce e o tratamento adequado da anquiloglossia e consequentemente a redução nas taxas de incidência de dificuldades de amamentação (SIMÃO R et al., 2008).

A frenotomia, conhecida como pique na língua, se dá através da secção do freio sem o eliminar e é uma técnica cirúrgica pouco invasiva, rápida, segura, com uso de anestesia local ou tópica e os custos para realizála são mínimos. As melhorias obtidas com o procedimento incluem melhora na pega do mamilo, diminuição da dor materna e manutenção da amamentação (MELO NSF, et al. 2011). A lei federal não elenca quais profissionais especificamente podem aplicar o teste da linguinha, ou seja, é permitido que diferentes profissionais realizem o mesmo, desde que tenham capacitação para isso. Entretanto, quando o objetivo é avaliar função estomatognática, é de fundamental importância que o diagnóstico realizado através do teste seja feito por uma equipe multiprofissional constituída por pediatra, cirurgião-dentista e fonoaudiólogo (MELO NSF et al., 2011). 


\section{CONCLUSÃO}

O percentual de gestantes atendidas nas Unidades Básicas de Saúde da Família (UBSF's) que não conhecem o teste da linguinha é alto. A grande maioria não recebeu orientação de nenhum profissional de saúde sobre a existência do teste, o que o mesmo detecta, período ideal e a importância de sua realização. Cabe às equipes das UBSF's orientar sobre a importância do teste da linguinha e o aleitamento materno de forma segura e tranquila durante o pré-natal. Com a realização do teste é possível diagnosticar e tratar precocemente a anquiloglossia, evitando desta maneira a amamentação inadequada e o desmame precoce.

\section{REFERÊNCIAS}

1. BRAGA LAS, et al. Prevalência de alteração no frênulo lingual e suas implicações na fala de escolares. Rev. CEFAC. 2009; $11(3):$ 378-390.

2. BRASIL. Ministério da Saúde. Secretaria de Atenção à Saúde. Departamento de Atenção Básica. Caderno de Atenção Básica. Atenção ao pré-natal de baixo risco 2013; Brasília: MS; 2013.

3. BRASIL. Presidência da República. Lei no 13002 de 20 de junho de 2014. Obriga a realização do protocolo de avaliação do frênulo da língua em bebês. Diário oficial da união, Poder Legislativo, Brasília, DF. 2014.

4. BRITO SF, et al. Frênulo lingual: classificação e conduta segundo ótica fonoaudiológica, odontológica e otorrinolaringológica. Rev. CEFAC. 2008; 10(3): 343-351.

5. CARVALHO CM, et al. Orientações no pré-natal: O que deve ser trabalhado pelos profissionais de saúde e a realidade encontrada. Rev. Gestão e Saúde. 2013; 4(2): 1988-2000.

6. CONSOLARO A. "Teste da linguinha" e a anquiloglossia: as controvérsias do assunto! Rev. Clín. Ortod. Dental Press. 2014; 13(1): 96-104.

7. GARCIA OJ, et al. Triagem neonatal ou teste do pezinho: conhecimento, orientações e importância para a saúde do recém-nascido. Rev. Cuidarte. 2008; 2(1):71-76.

8. GENARO KF, et al. Avaliação Miofuncional Orofacial - Protocolo MBGR. Rev. CEFAC. 2009; 11(2): $237-55$.

9. HOCHNADEL DB. Conhecimento das gestantes sobre a triagem auditiva neonatal. Monografia (Fonoaudiologia). Universidade Federal do Rio Grande do Sul, Porto Alegre, 2011; 37 p.

10. JARDIM, JB; HAHN, GV. Triagem auditiva neonatal: conhecimento das mães sobre o teste da orelhinha. Pediatr Mod, v. 50, p. 453-60, 2014.

11. MARTINELLI RLC, et al. Protocolo de avaliação do frênulo da língua em bebês. Rev. CEFAC. 2012; 1(14): $138-145$.

12. MARTINELLI RLC. Relação entre as características anatômicas do frênulo lingual e as funções de sucção e deglutição em bebês. Dissertação (Mestrado em Ciências). Universidade de São Paulo, Bauru, 2013; 112 p.

13. SILVA MBGM, LACERDA MR. "Teste do pezinho": por que coletar na alta hospitalar. Rev. Eletrônica de Enfermagem. 2003; 5(2):60-64.

14. MEDEIROS EB, RODRIGUES MJ. Conhecimento das gestantes sobre a saúde bucal de seu bebê. Rev. Assoc. Paul. Cir. Dent. 2003; 57(5): 381-386.

15. MELO NSF, et al. Anquiloglossia: relato de caso. Rev. SBO. 2011; 8(1): 102-107.

16. NASCIMENTO LS, et al. Teste da linguinha: Diagnóstico situacional sobre a aplicabilidade do protocolo em neonatos do distrito federal. Rev. Cefac. 2015; 17(6): 1889-1899.

17. PETERSON LJ, et al. Cirurgia Oral e Maxilofacial Contemporânea. Rio de Janeiro: Elsevier; 2009; $691 f$.

18. PIMENTEL IM. Adaptação cultural, linguística e psicométrica do protocolo de avaliação do frênulo da língua com escores para bebês. Dissertação (Mestrado em Terapia da Fala). Escola Superior de Saúde do Alcoitão, Alcabideche, Portugal, 2016; 64 p.

19. REIS DM, et al. Educação em saúde como estratégia de promoção de saúde bucal em gestantes. Rev. Ciência e Saúde Coletiva. 2010; 15(1): 269-276.

20. SIMÃO R, et al. Concepções de gestantes sobre a perda auditiva. Rev. Sallus. 2008; 2(1): 23-25.

21. SOARES CP, et al. Triagem Auditiva Neonatal: Aplicabilidade clínica na rotina dos médicos pediatras neonatologistas. Rev. CEFAC. 2008; 10(1): 110-116.

22. VENANCIO SI, et al. Anquiloglossia e aleitamento materno: Evidências sobre a magnitude do problema, protocolos de avaliação, segurança e eficácia da frenotomia. São Paulo: Instituto da saúde, 2015. 